\title{
Fully reconfigurable terahertz devices enabled by T-shaped graphene two-parallel-sheet
}

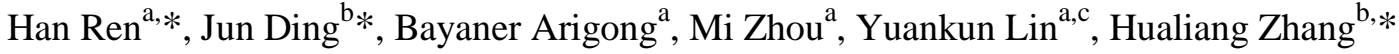 \\ ${ }^{a}$ Electrical Engineering Department, University of North Texas, 3940 N. Elm St., Denton, Texas 76207, USA \\ ${ }^{\mathbf{b}}$ Electrical and Computer Engineering Department, University of Massachusetts Lowell, Lowell, MA 01854, USA \\ ${ }^{\mathbf{c}}$ Physics Department, University of North Texas, Denton, Texas 76203, USA \\ *Corresponding authors. E-mail addresses: hanren@my.unt.edu (H. Ren), stevendingjun@gmail.com (J. Ding), \\ hualiang_zhang@uml.edu (H. Zhang)
}

\begin{abstract}
In this letter, we investigate fully reconfigurable terahertz (THz) devices enabled by a T-shaped graphene two-parallel-sheet (GTPS). The proposed T-shaped GTPS is constructed by two series-connected GTPS sections and one shunt-connected open-ended GTPS section. By applying electrical gating to the T-shaped GTPS, both electrical length and characteristic impedance of the terahertz waveguide (i.e. T-shaped GTPS) can be fully tuned. To demonstrate the tuning feature of the T-shaped GTPS, a variety of tunable THz devices are designed based on it, including reconfigurable tunable $\mathbf{T H z}$ transmission lines and a tunable THz coupler. The simulation results verify the large tuning range and multifunction of proposed devices.
\end{abstract}

Keywords - Reconfigurable; Terahertz; Graphene; Electrical gating.

\section{Introduction}

Graphene is an important two-dimensional (2D) material. It consists of 2-D arrangement of carbon atoms with a hexagonal structure [1]. Since its discovery in 2004 [2], it has been widely applied in various applications, such as chemistry, physics, and engineering. For example, graphene is a zero bandgap semiconductor which is very promising for nanoelectronic applications [3]. Graphene possesses a linear energy-momentum dispersion relation, which is similar with the dispersion relation of photons in free space [4]. This linear dispersion relation makes electrons behave like massless particles in graphene. As a result, graphene can obtain high carrier mobility $\left(5 \times 10^{5} \mathrm{~cm}^{2} / \mathrm{Vs}\right)$ [5]. Moreover, graphene's transport characteristics and conductivity can be tuned by varying its chemical potential (Fermi energy) [6]. To tune the chemical potential of graphene, methods using chemical doping, magnetostatic bias, and electrostatic bias have been applied and reported [7-14]. At the $\mathrm{THz}$ band, the monolayer graphene can realize unique surface plasmon polaritons (SPPs) which feature low loss, strong field confinement, and flexible reconfiguration among many other useful characteristics [15-16]. Due to these unique electronic properties, a lot of graphene-based devices have been proposed and designed in the past few years, including antennas [17-18], filters [19]-[20], switches [21-22], phase shifters [23], cloaks [24], absorbers
[25], modulators [26-27], oscillators [28], and transistors [29-30].

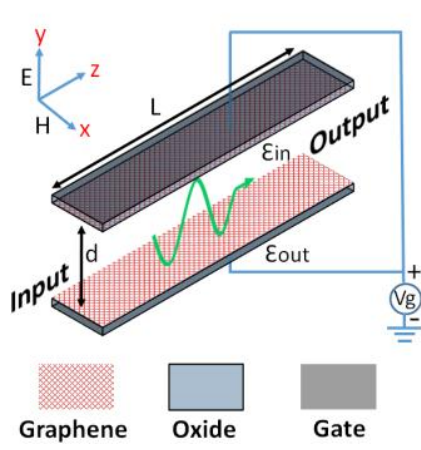

(a)

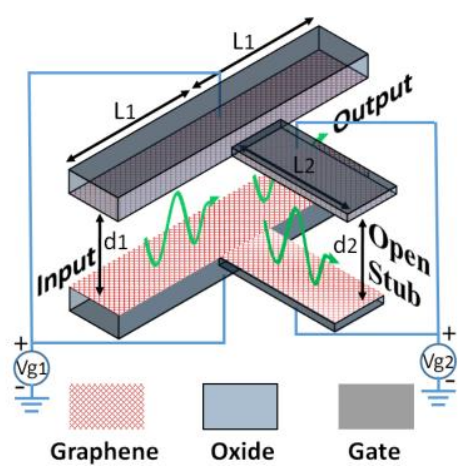

(b)
Fig. 1. Schematic diagram of (a) the graphene two-parallel-sheet (GTPS) with a single bias voltage and (b) the proposed T-shaped GTPS with two separated bias voltages.

Specifically, the so-called graphene two-parallel-sheet (GTPS) consisting of two identical graphene sheets separated by a spacing [31] has emerged as a promising graphene-based waveguide (for the guided propagation of electromagnetic waves). The GTPS features a dominant quasi-transverse electromagnetic mode, which has the similar attenuation as thicker-walled metal structures. By varying the chemical potentials of the GTPS's two graphene sheets, the surface conductivity on each graphene sheet can be tuned, leading to the tuning of the GTPS's propagation characteristics (e.g. phase shift). Based on this operating principle, several tunable $\mathrm{THz}$ components have been designed recently (e.g. phase shifters [23]). However, it is found that adjusting the chemical potentials of the gated sections within the GTPS will simultaneously change the characteristic impedance and the electrical length of the waveguide. As a result, it's difficult to keep one characteristic (e.g. impedance) while tuning the other characteristic (e.g. electrical length). This limitation has made it very difficult (if not impossible) to use the GTPS to realize functional tunable $\mathrm{THz}$ devices such as tunable multiple-port $\mathrm{THz}$ devices. To overcome this limitation, new GTPS structures need to be developed.

In this letter, a novel T-shaped GTPS is introduced to 
achieve independent control of its characteristic impedance and electrical length with a large tuning range. Following the theoretical analysis and to demonstrate the unique properties of the T-shaped GTPS, it is applied to design $\mathrm{THz}$ devices with various tunable characteristics (e.g. operating frequency, transmission spectra, and phase shift) and large tuning range. This letter is organized as follows: the theory and method will be described in Section 2. In Section 3, several designs of tunable terahertz devices based on the proposed design concept are presented and discussed. Finally, the conclusion is drawn in Section 4.

\section{Theory and method}

Fig. 1(a) shows the general schematic of the conventional GTPS with double-gate electrodes that are used to tune the chemical potential of the gated areas. The graphene sheets are separated with gates by oxide. Due to its waveguiding feature, it can be also called as graphene parallel-plate waveguide (GPPWG) [32]. Within the GTPS, two graphene sheets with horizontally infinite size are in parallel and separated with a distance of $d$. Based on the thin thickness, the graphene sheet has a 2D structure characterized by the surface conductivity $\sigma$, which can be derived using the Kubo formula [33]:

$$
\begin{aligned}
\sigma\left(\mu_{c}\right) & =\frac{i e^{2}\left(\omega-i \tau^{-1}\right)}{\pi \hbar^{2}} \times\left[\frac { 1 } { ( \omega - i \tau ^ { - 1 } ) ^ { 2 } } \int _ { 0 } ^ { \infty } \xi \left(\frac{\partial f_{d}\left(\xi, \mu_{c}, T\right)}{\partial \xi}-\right.\right. \\
\left.\frac{\partial f_{d}\left(-\xi, \mu_{c}, T\right)}{\partial \xi}\right) d \xi & \left.-\int_{0}^{\infty} \frac{f_{d}\left(-\xi, \mu_{c}, T\right)-f_{d}\left(\xi, \mu_{c}, T\right)}{\left(\omega-i \tau^{-1}\right)^{2}-4\left(\frac{\xi}{\hbar}\right)^{2}} d \xi\right]
\end{aligned}
$$

where $\mu_{c}$ is chemical potential, $\omega$ is angular frequency, $e$ is electron charge, $\tau$ is phenomenological electron relaxation time, $\hbar$ is reduced Planck's constant, and $\xi$ is energy. $f_{d}\left(\xi, \mu_{c}, T\right)$ is the Fermi-Dirac distribution:

$$
f_{d}\left(\xi, \mu_{c}, T\right)=\left(e^{\left(\xi-\mu_{c}\right) / k_{B} T}+1\right)^{-1}
$$

where $k_{B}$ is Boltzmann's constant, and $T$ is temperature. The graphene surface conductivity consists of intraband conductivity and interband conductivity, which dominate the first and second terms in Eq. (1) respectively. At the $\mathrm{THz}$ frequency range (the targeted frequency band in this letter) and below the interband transitiFoon threshold, the intraband contributions dominate the surface conductivity. Then Eq. (1) can be simplified as

$$
\sigma\left(\mu_{c}\right)=\sigma_{\text {intra }}=\frac{-i e^{2} k_{B} T}{\pi \hbar^{2}\left(\omega-i \tau^{-1}\right)}\left[\frac{\mu_{c}}{k_{B} T}+2 \ln \left(e^{-\mu_{c} / k_{B} T}+1\right)\right]
$$

Assuming $\mu_{c} / k_{B} T \gg 1$ over at least $500 \mathrm{~K}$ [32], the simplified equation of surface conductivity for all temperatures is then derived as:

$$
\sigma\left(\mu_{c}\right)=\frac{-i e^{2} \mu_{c}}{\pi \hbar^{2}\left(\omega-i \tau^{-1}\right)}
$$

In Fig. 1(a), the THz signal is assumed to propagate through the GTPS along the positive $z$-axis. The electromagnetic field

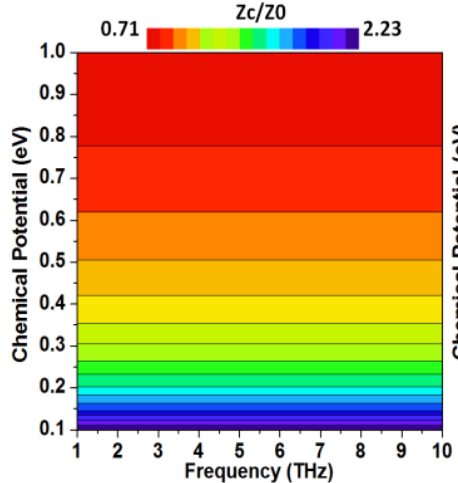

(a)

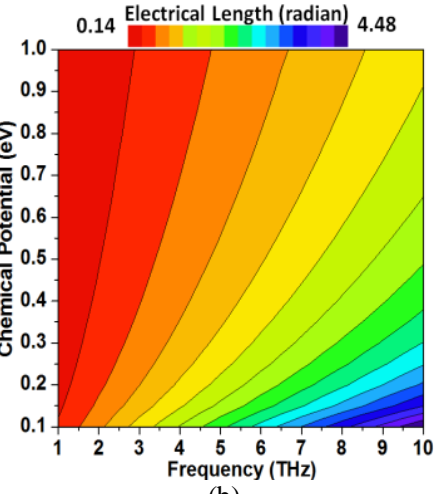

(b)
Fig. 2. Contours of (a) normalized characteristic impedance and (b) electrical length of the conventional GTPS as shown in Fig. 1(a), varying the operating frequency and chemical potential.

contains the transverse magnetic field (TM) and the transverse electric field (TE) along the $x$ - and $y$-axis, respectively. A quasi-TEM can be realized by the GTPS since it is similar to a perfectly metallic PPWG which supports a pure TEM mode [32]. The propagation constant $\beta$ is dominated by the magnetic field in TM waves, which is described as $H_{x}(y) e^{ \pm i \beta z}$. Assuming that the same surface conductivity is held in two graphene sheets, the approximate solution of $\beta$ in GTPS can be derived using Maxwell's equations [32]:

$$
\beta \simeq k_{0} \sqrt{\frac{\varepsilon_{\text {in }}}{\varepsilon_{0}}\left\{1+\frac{1}{\eta_{0}^{2} \sigma^{2}}\left[\frac{\varepsilon_{\text {in }}}{\varepsilon_{0}}-i \frac{2 \eta_{0} \sigma}{k_{0} d}\right]\right\}}
$$

where $\varepsilon_{\text {in }}$ is the permittivity of the material within the GTPS (between two graphene sheets, as shown in Fig. 1(a)), $k_{0}=\omega \sqrt{\mu_{0} \varepsilon_{0}}$ is the free-space wavenumber, and $\eta_{0}=$ $\sqrt{\mu_{0} / \varepsilon_{0}}$ is the free-space wave impedance. Due to sufficiently large surface conductivity, the permittivity of the material outside the waveguide plays a minor role on the propagation constant. To minimize the loss and effect from dielectric substrates [42-43], both inside and outside material's permittivities are set as free-space $\left(\varepsilon_{\text {in }}=\varepsilon_{\text {out }}=\varepsilon_{0}\right)$. Graphene with free-space can also achieve acceptable figure of merit (FoM) in a wide operating frequency band. Under these assumptions, Eq. (5) can be reduced as:

$$
\beta \simeq \sqrt{\omega^{2} \mu_{0} \varepsilon_{0}-i \frac{2 \varepsilon_{0} \omega}{\sigma d}}
$$

Substituting Eq. (4) into Eq. (6), the relationship between the propagation constant $\beta$ and the surface conductivity $\sigma$ is derived as follows:

$$
\beta \simeq \sqrt{\omega^{2} \mu_{0} \varepsilon_{0}+\frac{2 \omega^{2} \varepsilon_{0} \pi \hbar^{2}}{e^{2} \mu_{c} d}-i \frac{2 \omega \varepsilon_{0} \pi \hbar^{2}}{e^{2} \mu_{c} d \tau}}
$$

where the relaxation time $\tau$ is set as $4 \mathrm{ps}$. At the $\mathrm{THz}$ frequency 
range, the real part of the propagation constant plays the major role. Based on the transfer-matrix method and the full-wave simulation [34-35], the electrical length $\theta_{c}$ and the characteristic impedance $Z_{c}$ of the GTPS-based $\mathrm{THz}$ waveguide can be derived as follows:

$$
\begin{aligned}
& \theta_{c}=\beta L \\
& Z_{c}=\frac{E_{y} d}{H_{x}}=\frac{\beta d}{\omega \varepsilon_{0}}
\end{aligned}
$$

where $L$ is the physical length of GTPS. From Eqs. (8) - (9), it is clear that the characteristics of GTPS-based waveguides are dominated by the propagation constant. Meanwhile, the propagation constant can be tuned by the chemical potential using Eq. (7). This is the key feature of GTPS for terahertz component design. Fig. 2 shows the calculation results of the normalized characteristic impedance and electrical length of the GTPS-based waveguide by varying the operating frequency and the chemical potential from Eqs. (7) - (9) (physical length $L$ and spacing distance $d$ are set to 1 um and $300 \mathrm{~nm}$, respectively). Throughout this letter, $Z_{0}$ is set as the free-space impedance of the waveguide with $d=300 \mathrm{~nm}$.

In our design, the realizable range of chemical potential $\mu_{c}$ is from 0 to $1 \mathrm{eV}$. To tune the chemical potential, one of the efficient approaches is to add the bias voltage $V_{g}$ on the top and bottom graphene sheets simultaneously (as shown in Fig. 1(a)). The variation of chemical potential is equivalent to the voltage drop $V_{c h}$ across the quantum capacitance $C_{q}$. Based on the Kirchhoff's law, $V_{c h}$ can be derived as [36-37]:

$$
V_{c h}=\frac{\mu_{c}}{e}=\frac{C_{o x} V_{g}}{C_{o x}+\frac{1}{2} C_{q}\left(V_{c h}\right)}
$$

where $C_{o x}=\varepsilon_{o x} / t_{o x}\left(\varepsilon_{o x}\right.$ and $t_{o x}$ are the permittivity and thickness of the gate oxide, respectively) is the oxide capacitance. Under the condition $e V_{c h} \gg k_{B} T$, a simple equation of $C_{q}$ can be expressed as:

$$
C_{q}=\frac{2 e^{3}\left|V_{c h}\right|}{\pi\left(\hbar v_{F}\right)^{2}}
$$

where $v_{F}$ is Fermi velocity. Substituting Eq. (11) into Eq. (10) yields:

$$
\mu_{c}=\frac{\hbar v_{F}}{2 e^{2}}\left(-\pi C_{o x} \hbar v_{F}+\sqrt{\left(\pi C_{o x} \hbar v_{F}\right)^{2}+4 e^{3} \pi C_{o x} V_{g}}\right)
$$

As a result, the GTPS-based waveguide can be controlled by tuning the bias voltage on the top and bottom graphene sheets. By Eq. (12), appropriate permittivity and thickness of the oxide can set the optimal range of the bias voltage for practical issues such as the breakdown of the oxide [44]. However, as mentioned previously, there is a strong constraint for the realizable propagation characteristics using the conventional GTPS. From Eqs. (8) - (9), it is found that a given propagation constant will lead to a certain combination of electrical length and characteristics impedance. This property will prevent the
GTPS from achieving the full-scale tuning on the waveguide's characteristics (e.g. characteristic impedance and electrical length). Therefore, it is very difficult to achieve flexible

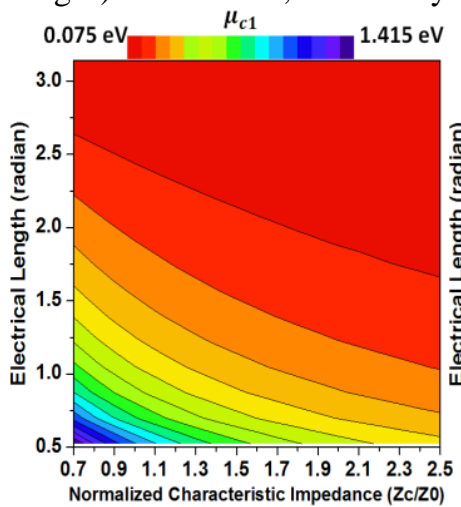

(a)

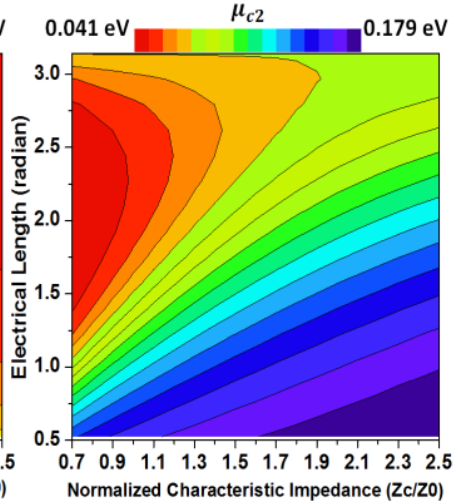

(b)
Fig. 3. Calculated results for the proposed T-shaped GTPS: (a) Contours of normalized characteristic impedance and electrical length of the series section in the GTPS with a chemical potential $\mu_{c 1}$; (b) Contours of normalized characteristic impedance and electrical length of the open-ended section in the GTPS with a chemical potential $\mu_{c 2}$.

combination of electrical length and characteristics impedance using the structure in Fig. 1(a).

To address this issue, a new T-type GTPS as shown in Fig. 1 (b) is proposed. This structure is constructed by two series-connected GTPS sections and one shunt-connected open-ended GTPS section. The two series-connected GTPS sections have identical physical dimensions (length $L 1$ and thickness $d 1$ ) and are gated by the same bias voltage $V_{g 1}$. An additional shunt-connected open-ended GTPS (length L2, thickness $d 2$, and bias voltage $V_{g 2}$ ) is tapped at the center of these two series sections. To avoid undesired influence on graphene plasmons, the width of all GTPSs should be larger than $20 \mathrm{~nm}$ [38-39]. Different thicknesses of oxides are also set to separate two unique bias voltages. The proposed T-type structure can be analyzed using the transfer matrix method (i.e. ABCD matrix) [40]:

$$
\begin{aligned}
& A_{T}=D_{T}=\cos 2 \theta_{1}-\frac{Z_{1} \sin 2 \theta_{1} \tan \theta_{2}}{2 Z_{2}} \\
& B_{T}=i Z_{1} \sin 2 \theta_{1}-i \frac{Z_{1}{ }^{2} \sin ^{2} \theta_{1} \tan \theta_{2}}{Z_{2}} \\
& C_{T}=i \frac{2 \sin \theta_{1} \cos \theta_{2}}{Z_{1}}+i \frac{\cos ^{2} \theta_{1} \tan \theta_{2}}{Z_{2}}
\end{aligned}
$$

where $Z_{1}, Z_{2}$ and $\theta_{1}, \theta_{2}$ are the characteristic impedances and the electrical lengths of the series and open-ended sections in the T-shaped GTPS, respectively. To realize the specific values of impedance $Z_{c}$ and electrical length $\theta_{c}$ using the proposed T-type structure, $A_{T}=\cos \theta_{c}$ and $B_{T}=i Z_{c} \sin \theta_{c}$ should be satisfied. Substituting these two equations into Eqs. (13) - (14), yields:

$$
Z_{1}=\frac{Z_{c} \sin \theta_{c} \cot \theta_{1}}{1+\cos \theta_{c}}
$$




$$
Z_{2}=\frac{Z_{c} \sin \theta_{c} \cos ^{2} \theta_{1} \tan \theta_{2}}{\left(\cos 2 \theta_{1}-\cos \theta_{c}\right)\left(1+\cos \theta_{c}\right)}
$$

Based on Eqs. (8) - (9), it is found that the characteristic impedance and the electrical length of each section in the T-shaped GTPS can be controlled by the corresponding propagation constant. By substituting Eqs. (8) - (9) into Eq. (16) - (17), two important design equations can be obtained:

$$
\begin{aligned}
& \frac{Z_{c} \sin \theta_{c} \cot \left(\beta_{1} L_{1}\right)}{1+\cos \theta_{c}}=\frac{\beta_{1} d_{1}}{\omega \varepsilon_{0}} \\
& \frac{Z_{c} \sin \theta_{c} \cos ^{2}\left(\beta_{1} L_{1}\right) \tan \left(\beta_{2} L_{2}\right)}{\left(\cos \left(2 \beta_{1} L_{1}\right)-\cos \theta_{c}\right)\left(1+\cos \theta_{c}\right)}=\frac{\beta_{2} d_{2}}{\omega \varepsilon_{0}}
\end{aligned}
$$

where $\beta_{1}$ and $\beta_{2}$ are the propagation constants of the series-connected and shunt open-ended GTPSs, respectively. It is found that for a given combination of impedance $Z_{c}$ and electrical length $\theta_{c}$, the corresponding propagation constants $\beta_{1}$ and $\beta_{2}$ can be obtained by solving Eqs. (18) - (19). Therefore, by tuning the chemical potentials of the series-connected and shunt-connected GTPS sections, full-scale tunabilitiy can be achieved by using the proposed T-shaped GTPS. This is validated by Fig. 3, which shows that the values of normalized characteristic impedance and electrical length of the series-connected and shunt-connected GTPS sections can be flexible by tuning the chemical potential $\mu_{c 1}, \mu_{c 2}$ respectively (here, we assume that the operating frequency $f=5 \mathrm{THz}, d 1=d 2=200 \mathrm{~nm}, L 1=500 \mathrm{~nm}$, and $L 2=1$ um). In summary, the design procedure for the T-shaped GTPS to achieve the desired characteristic impedance and electrical length can be described as the following:

1) Select the desired values of characteristic impedance $Z_{c}$ and electrical length $\theta_{c}$, which will be realized by the T-shaped GTPS structure;

2) Use Eq. (18) to solve the value of $\beta_{1}$, then obtain the value of $\beta_{2}$ by Eq. (19);

3) Substitute $\beta_{1}$ and $\beta_{2}$ into Eq. (7) to get the values of $\mu_{c 1}$ and $\mu_{c 2}$;

4) Finally, the corresponding bias voltages $V_{g 1}$ and $V_{g 2}$ can be obtained by solving Eq. (12).

In the next section, several tunable $\mathrm{THz}$ devices using the proposed T-shaped GTPS are designed and simulated to demonstrate its unique features. The simulation results are obtain by the full-wave electromagnetic simulator ANSYS HFSS.

\section{Designs and results}

\subsection{Terahertz transmission line with tunable phase or tunable operating frequency}

Transmission line is an indispensable component in the high-frequency electronic systems for applications such as telecommunication and radar. The conventional transmission line employs single section with the characteristic impedance $Z_{c}$ and the electrical length $\theta_{c}$ (as shown in Fig. 4(a)). In general, it is very difficult to tune its characteristic impedance and the electrical length simultaneously (e.g. varying the phase shift while the impedance matching can be maintained).

To achieve a tunable $\mathrm{THz}$ transmission line with tunable phase response and a fixed characteristic impedance, the

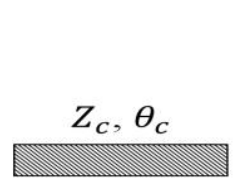

(a)

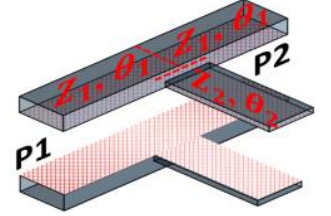

(b)
Fig. 4. Configuration of (a) the conventional transmission line and (b) the proposed tunable transmission line using the T-shaped GTPS.

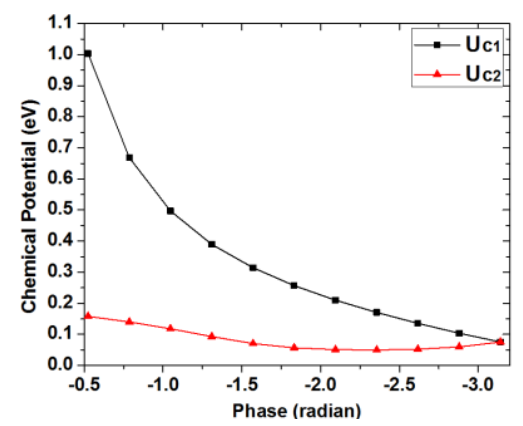

Fig. 5. Values of the chemical potentials $\mu_{c 1}$ and $\mu_{c 2}$ when the phase of the proposed $\mathrm{THz}$ transmission line is tuned to be different values.

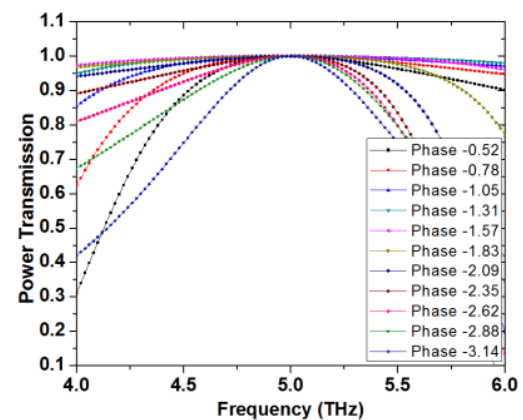

(a)

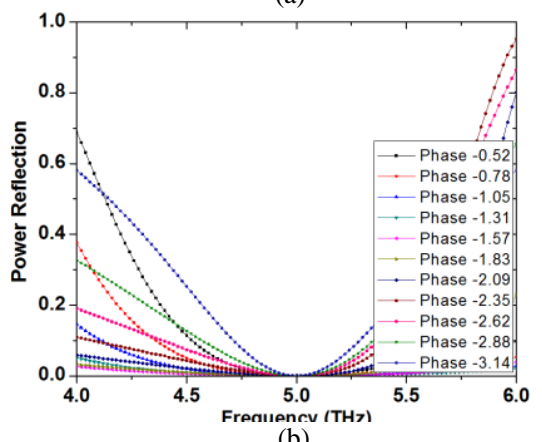

(b) 


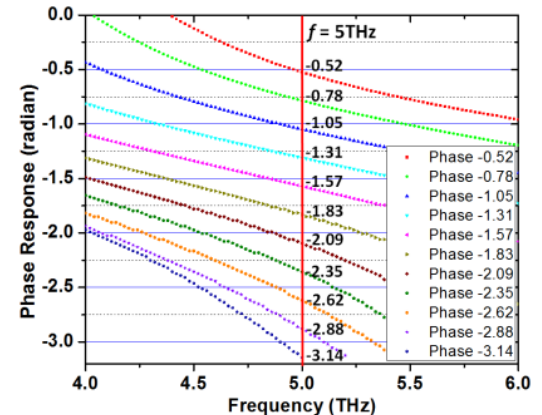

(c)

Fig. 6. Simulation results of (a) power transmission $\left(\mathrm{S}_{21}\right)$, (b) power reflection (S11), and (c) phase response of the proposed $\mathrm{THz}$ transmission line with tunable phase response.

proposed T-shaped GTPS (as shown in Fig. 4(b)) is applied to replace the conventional structure. In the proposed design, the operating frequency is $f=5 \mathrm{THz}$, the physical lengths of the T-shaped GTPS are $L 1=500 \mathrm{~nm}, L 2=1 \mathrm{um}$, and the thickness of the GTPS are $d 1=d 2=200 \mathrm{~nm}$ (referring to Fig. 1(b)). The tuning range of the phase $\theta_{c}$ is from -0.52 to -3.14 , and the characteristic impedance $Z_{c}$ is fixed to be equal to the input/output port impedance $\left(Z_{0}\right)$. Following the design procedure described in last section, the chemical potentials $\mu_{c 1}$, $\mu_{c 2}$ corresponding to different phases can be obtained, which are plotted in Fig. 5. Fig. 6 presents the simulation results of scattering parameters (S-parameters) and phase responses of the proposed transmission line. From Fig. 6(a), it is observed that complete power transmission $\left(\left|\mathbf{S}_{21}\right|=1\right)$ is achieved at the operating frequency $5 \mathrm{THz}$. For the power reflection, it can be smaller than $0.00001\left(\left|\mathrm{~S}_{11}\right|<0.00001\right.$, as shown in Fig. 6(b)) at the working frequency, which indicates that the transmission line is always matched to the input/output port impedance $\left(Z_{0}\right)$ (therefore its characteristic impedance is fixed to be $Z_{0}$ ). The phase responses are presented in Fig. 6(c). It is observed that, by tuning the chemical potential to the appropriate values (as shown in Fig. 5), the phase of the proposed THz transmission line can be tuned from -0.52 to -3.14 . Based on our analysis, the tuning range of the realizable phase using the proposed design can be further extended, if a larger chemical potential is available in practice (e.g. $\mu_{c}>1 \mathrm{eV}$ ).

Furthermore, it is found that the proposed design as shown in Fig. 4(b) can be also employed to achieve a tunable transmission line with tunable operating frequencies and a fixed phase and characteristic impedance. To verify this feature, a one-eighth wavelength $\mathrm{THz}$ transmission line with tunable operating frequency from $3 \mathrm{THz}$ to $7 \mathrm{THz}$ is designed and simulated. The physical dimensions of the series/open-end GTPSs applied in the design are: $L 1=500 \mathrm{~nm}, L 2=1 \mathrm{um}$, and $d 1=d_{2}=200 \mathrm{~nm}$. The input/output port impedance is again equal to $Z_{0}$. By solving Eqs. (18) - (19) and Eq. (7), the corresponding values of chemical potentials $\mu_{c 1}, \mu_{c 2}$ (when the transmission line is operating at different frequencies) are given in Table 1. Fig. 7(a) shows that the desired complete power transmission $(|\mathbf{S} 21|=1)$ is achieved at each operating frequency (e.g. 3, 4, 5, 6, 7 THz). In Fig. 7(b), extremely low power reflection is obtained at desired operating frequencies (it is again tuned from 3 to $7 \mathrm{THz}$ ), verifying the good matching of the transmission line. For the phase response, the induced phase response is kept as -0.785 while the operating frequency is tuned from $3 \mathrm{THz}$ to $7 \mathrm{THz}$ (as shown in Fig. 7(c)).

\subsection{Terahertz couplers with tunable phase difference}

Next, THz couplers with reconfigurable responses will be demonstrated. Coupler is one of the fundamental high-frequency circuit components. It has been applied extensively in various high frequency systems including data modulators, multipliers, and antenna feeding networks. Fig. 8(a) shows the configuration of a conventional coupler with fixed phase difference [41]. The phase difference $\Delta \theta$ between two output ports $\mathrm{P} 3$ and $\mathrm{P} 4$ can be characterized as:

Table 1. Calculated values of chemical potentials for the T-shaped GTPS-based transmission line with tunable operating frequency.

\begin{tabular}{|c|c|c|}
\hline $\boldsymbol{f}(\mathbf{T H z})$ & $\boldsymbol{\mu}_{\boldsymbol{c 1}}(\mathrm{eV})$ & $\boldsymbol{\mu}_{\boldsymbol{c} \mathbf{2}}(\mathbf{e V})$ \\
\hline $\mathbf{3}$ & 0.384 & 0.034 \\
\hline $\mathbf{4}$ & 0.523 & 0.079 \\
\hline $\mathbf{5}$ & 0.668 & 0.140 \\
\hline $\mathbf{6}$ & 0.819 & 0.215 \\
\hline $\mathbf{7}$ & 0.977 & 0.307 \\
\hline
\end{tabular}
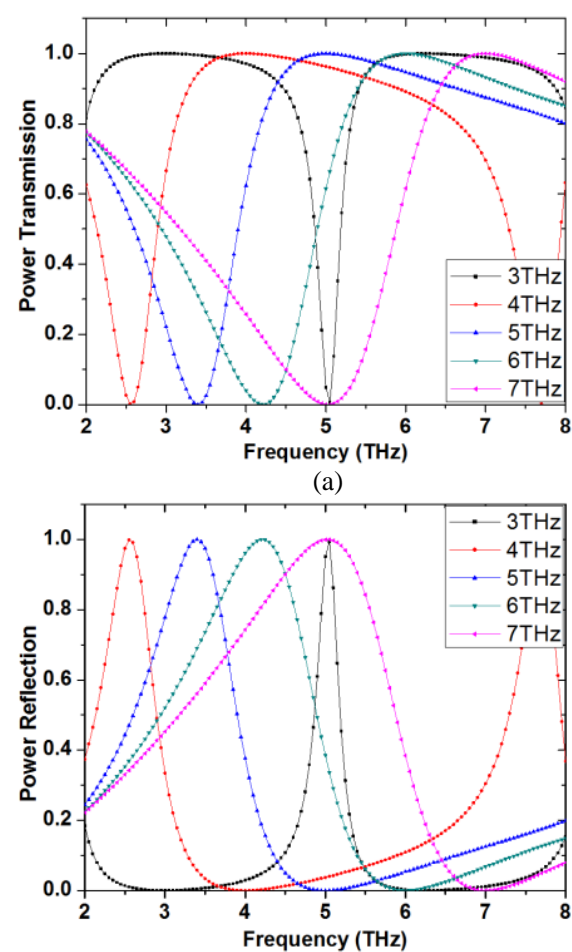

(b)

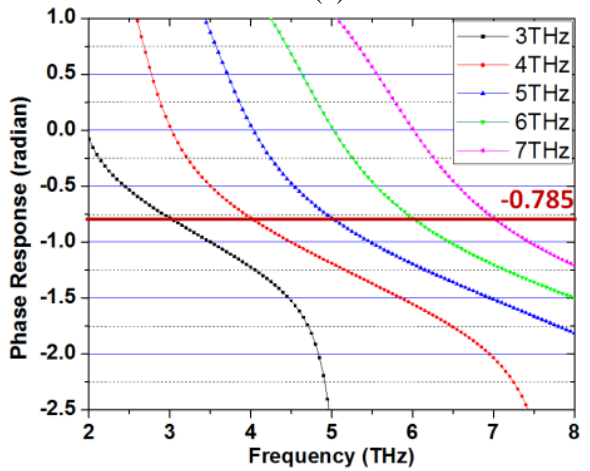


(c)

Fig. 7. Simulation results of (a) power transmission (S21), (b) power reflection (S11), and (c) phase response of the proposed $\mathrm{THz}$ transmission line with tunable operating frequency.

$$
\begin{aligned}
& P 41-P 31=-\Delta \theta \\
& P 32-P 42=\pi+\Delta \theta
\end{aligned}
$$

The characteristic impedances and electrical lengths of each transmission line section within the coupler can be derived as:

$$
\begin{aligned}
& Z_{a}=Z_{0} \sin \Delta \theta \\
& Z_{b}=Z_{c}=\frac{Z_{0} \sin \Delta \theta}{\sqrt{1+\sin ^{2} \Delta \theta}} \\
& \theta_{a}=\pi / 2 \\
& \theta_{b}=\cos ^{-1}\left(\frac{-\cos \Delta \theta}{\sqrt{2}}\right) \\
& \theta_{c}=\pi-\theta_{b}
\end{aligned}
$$

where $Z_{0}$ is the input/output port impedance. To realize a coupler with tunable phase difference (between output ports), the proposed T-shaped GTPS (as shown in Fig. 1(b)) is applied to replace each transmission line section of the coupler in Fig. 8(a). The general schematic of the designed tunable $\mathrm{THz}$ coupler is shown in Fig. 8(b). The design procedure of it can be summarized as the following: 1) determine the range of the phase difference $\Delta \theta$ between the output ports; 2) use Eqs. (22) (26) to obtain the values of the characteristic impedance and electrical length of each transmission line section; 3 ) follow the design procedures mentioned in Section 2 to get the values of chemical potentials for different GTPS sections. To verify the proposed design concept, a coupler working at $3 \mathrm{THz}$ with the phase difference (between two output ports P3 and P4) tuned from 0.35 to 2.8 is designed and simulated. For the $T_{a}$ sections (as marked in Fig. 8(b)), $L 1=800 \mathrm{~nm}, L_{2}=1 \mathrm{um}$, and $d_{1}=d_{2}=$ $300 \mathrm{~nm}$. For the $T_{b}$ and $T_{c}$ sections, $L 1=500 \mathrm{~nm}, L 2=1 \mathrm{um}$, and $d 1=d 2=100 \mathrm{~nm}$. Fig. 9 shows the calculated values of chemical potentials for each $\mathrm{T}$ section when the phase difference is varied. Fig. 10 shows the simulation results of the coupler with the phase difference $\Delta \theta=0.52$. From Fig. 10(a), at the operating frequency $3 \mathrm{THz}$, the power transmission of the designed coupler are $0.5(|\mathrm{~S} 31|,|\mathrm{S} 41|,|\mathrm{S} 32|,|\mathrm{S} 42|=0.5)$. The power reflection and isolation are smaller than 0.00001 (|S11|, $|\mathrm{S} 21|<0.00001)$. In Fig. 10(b), the desired phase difference between the two output ports is achieved $(P 41-P 31=-0.52$, and $P 32-P 42=-2.62$ at $3 \mathrm{THz})$. In Fig. 11, the simulation results of the coupler with the phase difference reconfigured to $\Delta \theta=0.78$ are plotted. At $3 \mathrm{THz}$, the power transmission are about 0.5 while the power reflection and isolation are both less than 0.00001 (as shown in Fig. 11(a)). In Fig. 11(b), the desired phase responses are achieved $(P 41-P 31=-0.78$, and $P 32-$ $P 42=-2.35$ at $3 \mathrm{THz}$ ).
Overall, all the simulation results agree well with the design theory, verifying the flexible performance of the designed tunable THz devices based on the proposed T-shaped GTPS. Due to the large tuning range of the proposed devices, they can lead to significant reductions in both size and cost of $\mathrm{THz}$ systems.

\section{Conclusion}

In conclusion, we have investigated several new reconfigurable terahertz $(\mathrm{THz})$ devices based on a $\mathrm{T}$-shaped graphene two-parallel-sheet (GTPS). Enabled by the T-shaped structure, the characteristic impedance and electrical length of the resulting $\mathrm{THz}$ waveguide can be independently controlled. To verify the proposed design concept, several designs of tunable
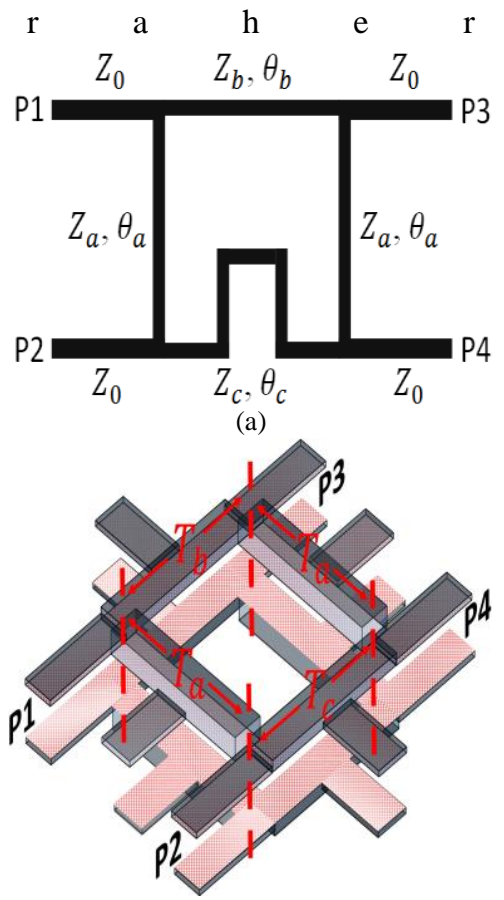

(b)

Fig. 8. Configuration of (a) the conventional coupler with fixed phase difference between output ports (top view) and (b) the proposed coupler with tunable phase difference (3D view), consisting of T-shaped GTPS sections $T a$, $T b$, and $T c$.

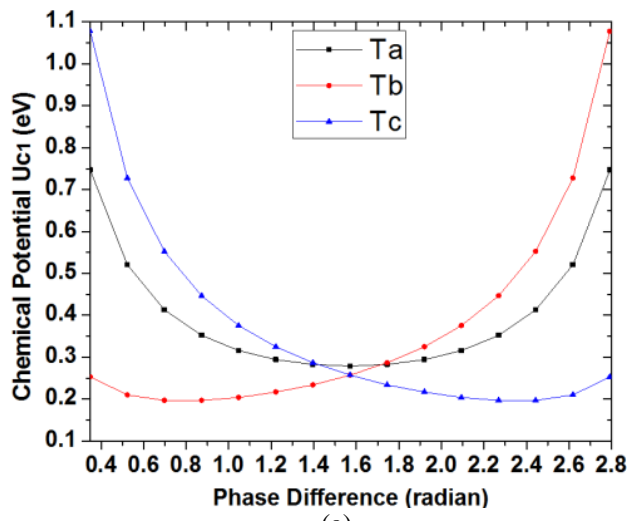

(a) 


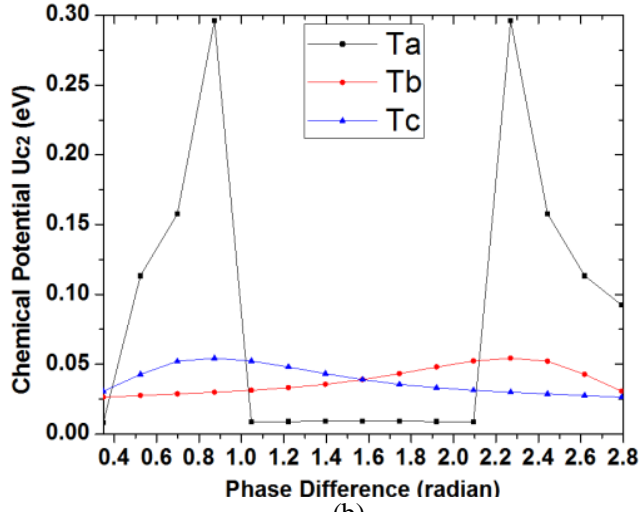

(b)

Fig. 9. Calculated chemical potentials (a) $\mu_{c 1}$ and (b) $\mu_{c 2}$ for each section (i.e. $\left.T_{a}, T_{b}, T_{c}\right)$ in the proposed tunable $\mathrm{THz}$ coupler, where the phase difference $\Delta \theta$ varies from 0.35 to 2.8 .

devices, including transmission lines with tunable phase or tunable operating frequency and a coupler with tunable phase

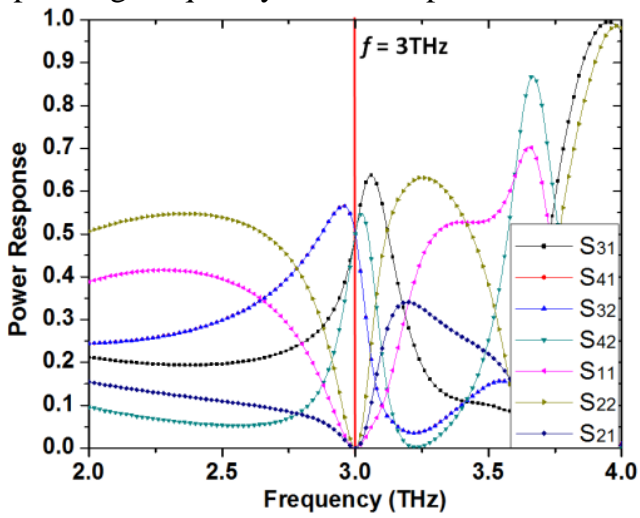

(a)

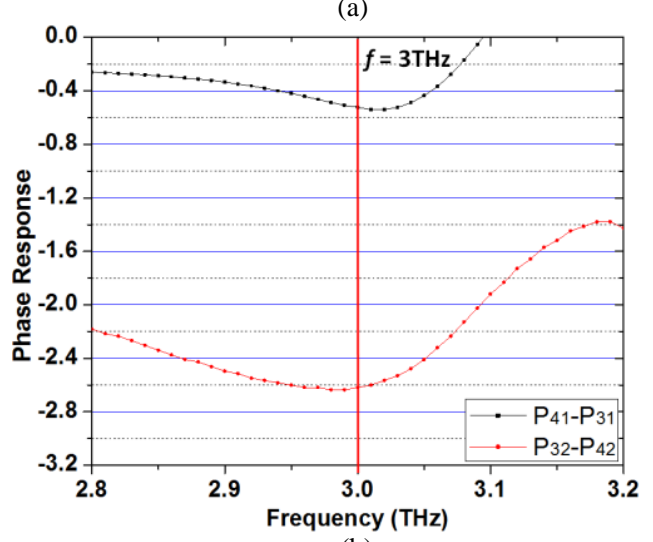

(b)

Fig. 10. Simulation results of (a) power response and (b) phase response of the proposed tunable $\mathrm{THz}$ coupler when $\Delta \theta=0.52$.

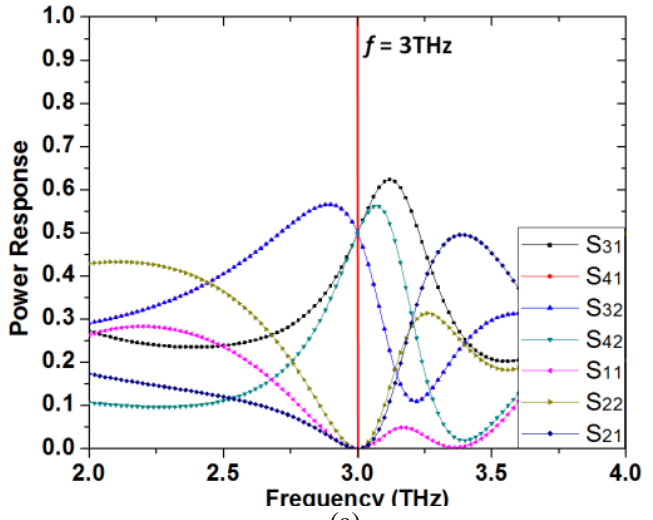

(a)

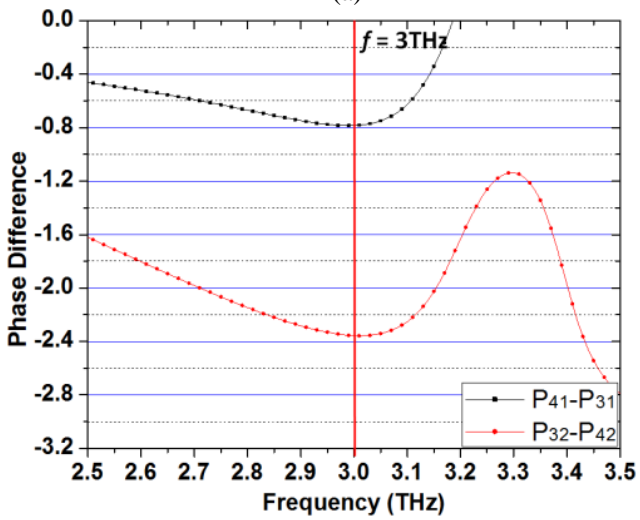

(b)

Fig. 11. Simulation results of (a) power response and (b) phase response of the proposed tunable $\mathrm{THz}$ coupler when $\Delta \theta=0.78$.

difference are designed and simulated. All the simulation results show good agreement with the design theory. The proposed graphene-based structure provides a novel approach to realize terahertz components with tunable and flexible responses, demonstrating the potential of graphene for terahertz systems and applications.

\section{Conclusion}

In conclusion, we have investigated several new reconfigurable terahertz $(\mathrm{THz})$ devices based on a T-shaped graphene two-parallel-sheet (GTPS). Enabled by the T-shaped structure, the characteristic impedance and electrical length of the resulting $\mathrm{THz}$ waveguide can be independently controlled. To verify the proposed design concept, several designs of tunable terahertz devices, including transmission lines with tunable phase or tunable operating frequency and a coupler with tunable phase difference are designed and simulated. All the simulation results show good agreement with the design theory. The proposed graphene-based structure provides a novel approach to realize terahertz components with tunable and flexible responses, demonstrating the potential of graphene for terahertz systems and applications.

\section{Acknowledgements}

This work is supported by research grants from the U.S. National Science Foundation under Grant Nos. ECCS-1550741, and CMMI-1266251. 


\section{References}

[1] A. K. Geim and K. S. Novoselov, "The rise of graphene," Nat. Mater., vol. 6, pp. 183-191, 2007.

[2] K. S. Novoselov, A. K. Geim, S. V. Morozor, D. Jiang, Y. Zhang, S. V. Dubonos, I. V. Grigorieva, and A. A. Firsov, "Electric field effect in atomically thin carbon films," Science, vol. 306, no. 5696, pp. 666-669, Oct. 2004.

[3] A. H. C. Neto, F. Guinea, N. M. R. Peres, K. S. Novoselov, and A. K. Geim, "The electronic properties of graphene," Rev. Mod. Phys., vol. 81, no. 1, pp. 109-162, Jan. 2009.

[4] A. S. Mayorov, R. V. Gorbachev, S. V. Morozov, L. Britnell, R. Jalil, L. A. Ponomarenko, P. Blake, K. S. Novoselov, K. Watanabe, T. Taniguchi, and A. K. Geim, "Micrometer-scale ballistic transport in encapsulated graphene at room temperature," Nano Lett., vol. 11, no. 6, pp. 2396-2399, May 2011.

[5] H. S. P. Wong, and D. J. Akinwande, Carbon Nanotube and Graphene Device Physics, Cambridge University Press: Cambridge, 2011

[6] L. Jiang, and Y. Zheng, "Remarkable enhancement of terahertz conductivity of graphene tuned by periodic gate voltage," Phys. Lett. A, vol. 375, pp. 203-207, Oct. 2010.

[7] Y. Zhang, Y. -W. Tan, H. L. Stormer, and P. Kim, "Experimental observation of the quantum Hall effect and Berry's phase in graphene," Nature, vol. 438, pp. 201-204, Nov. 2005.

[8] Z. Q. Li, E. A. Henriksen, Z. Jiang, Z. Hao, M. C. Martin, P. Kim, H. L. Stormer, D. N. Basov, "Dirac charge dynamics in graphene by infrared spectroscopy," Nat. Phys., vol. 4, pp. 532-535, Jun. 2008.

[9] A. Vakil and N. Engheta, "Transformation optics using graphene," Science, vol. 332, no. 6035, pp. 1291-1294, Jun. 2011.

[10] G. W. Hanson, "Dyadic Green's functions for an anisotropic, non-local model of biased graphene," IEEE Trans. Antenna Propag., vol. 56, no. 3 pp. 747-757, Mar. 2008.

[11] P. Y. Chen and A. Alù, "Atomically thin surface cloak using graphene monolayers," ACS Nano, vol. 5, no. 7, pp. 5855-5863, Jun. 2011.

[12] V. P. Gusynin, S. G. Sharapov, and J. P. Carbotte, "Magneto-optical conductivity in graphene," J. Phys.: Cond. Mater., vol. 19, no. 2, pp. 026222-026222, Jan. 2007

[13] X. Chen, Z. Shi, S. Zhang, K. Song, and G. Zhou, "Anti-bias voltage electron-Kondo transport in a quantum dot device driven by a graphene sheet," Phys. Lett. A, vol. 379, pp. 187-191, Nov. 2014.

[14] A. Belouad, A. Jellal, and Y. Zahidi, "Gate-tunable graphene quantum dot and Dirac oscillator," Phys. Lett. A, vol. 380, pp. 773-778, Dec. 2015.

[15] F. Rana, "Graphene terahertz plasmon oscillators," IEEE Trans. Nanotechnol., vol. 7, no. 1, pp. 91-99, Jan. 2008.

[16] P. Y. Chen and A. Alù, "Terahertz metamaterial devices based on graphene nanostructures," IEEE Trans. Terahertz Sci. Technol., vol. 3, no. 6, pp. 748-756, Nov. 2013.

[17] A. Cabellos, I. Llatser, E. Alarcón, A. Hsu, and T. Palacios, "Use of THz photoconductive sources to characterize tunable graphene RF plasmonic antennas," IEEE Trans. Nanotechnol., vol. 14, no. 2, pp. 390-396, Mar. 2015.

[18] X. C. Wang, W. S. Zhao, J. Hu, and W. Y. Yin, "Reconfigurable terahertz leaky-wave antenna using graphene-based high-impedance surface," IEEE Trans. Nanotechnol., vol. 14, no. 1, pp. 62-69, Jan. 2015.

[19] D. Correas-Serrano, J. S. Gomez-Diaz, J. Perruisseau-Carrier, and A. Álvarez-Melcón, "Graphene-based plasmonic tunable low-pass filters in the terahertz band," IEEE Trans. Nanotechnol., vol. 13, no. 6, pp. 1145-1153, Nov. 2014

[20] H. Deng, Y. Yan, and Y. Yu, "Tunable flat-top bandpass filter based on coupled resonators on a graphene sheet," IEEE Photon. Technol. Lett., vol. 27, no. 11, pp. 1161-1164, Jun. 2015.

[21] Y. Huang, L. S. Wu, M. Tang, and J. Mao, "Design of a beam reconfigurable $\mathrm{THz}$ antenna with graphene-based switchable high-impedance surface," IEEE Trans. Nanotechnol., vol. 13, no. 1, pp. 70-79, Jan. 2014

[22] P. Sharma, J. P. Carrier, C. Moldovan, and A. M. Ionescu, "Electromagnetic performance of RF NEMS graphene capacitive switches," IEEE Trans. Nanotechnol., vol. 11, no. 4, pp. 836-842, Jul. 2012.

[23] P. Y. Chen, C. Argyropoulos, and A. Alù, "Terahertz antenna phase shifters using integrally-gated graphene transmission-lines," IEEE Trans. Antennas Propag., vol. 61, no. 4, pp. 1528-1537, Apr. 2013.
[24] P. Y. Chen, J. Soric, Y. R. Padooru, H. M. Bernety, A. B. Yakovlev, and A. Alù, "Nanostructured graphene metasurface for tunable terahertz cloaking," New J. Phys., vol. 15, pp. 123029-123029, Dec. 2013.

[25] Z. Fang, Y. Wang, A. E. Schlather, Z. Liu, P. M. Ajayan, F. J. García de Abajo, P. Nordlander, X. Zhu, and N. J. Halas, "Active tunable absorption enhancement with graphene nanodisk arrays," Nano Lett., vol. 14, no. 1, pp. 299-304, 2014.

[26] M. Liu, X. Yin, E. Ulin-Avila, B. Geng, T. Zentgraf, L. Ju, F. Wang, and X. Zhang, "A graphene-based broadband optical modulator," Nature, vol. 474, pp. 64-67, Jun. 2011.

[27] M. Liu, X. Yin, and X. Zhang, "Double-layer graphene optical modulator," Nano Lett., vol. 12, no. 3, pp. 1482-1485, Feb. 2012.

[28] J. Fu, B. Lv, R. Li, R. Ma, W. Chen, and F. Meng, "Excitation of graphene plasmons as an analogy with the two-level system," Phys. Lett. A, vol. 380, pp. 803-808, Dec. 2015.

[29] C. Bai, J. Wang, and Y. Yang, "Anisotropic pairing symmetry effect on crossed Andreev reflection in a graphene-based transistor," Phys. Lett. A, vol. 375, pp. 1023-1027, Jan. 2011.

[30] M. Gupta, N. Gaur, P. Kumar, S. Singh, N. K. Jaiswal, and P.N. Kondekar, "Tailoring the electronic properties of a Z-shaped graphene field effect transistor via B/N doping," Phys. Lett. A, vol. 379, pp. 710-718, Dec. 2014.

[31] G. W. Hanson, "Quasi-transverse electromagnetic modes supported by a graphene parallel-plate waveguide," J. Appl. Phys., vol. 104, pp. 084314-084314, Oct. 2008.

[32] H. Ren, J. Ding, B. Arigong, M. Zhou, J. Shao, Y. Lin, and H. Zhang, "A novel design of tunable terahertz devices using graphene parallel-plate waveguide," in Proc. of 2015 IEEE AP Symposium, Vancouver, Canada, 19-25 July 2015, pp. 2413-2414.

[33] M. Dressel, and G. Grüner, Electrodynamics of Solids, Cambridge University Press: London, 2002.

[34] S. E. Kocabas, G. Veronis, D. A. B. Miller, and S. Fan, "Transmission line and equivalent circuit models for plasmonic waveguide components," IEEE Quan. Electron., vol. 14, no. 6, pp. 1462-1472, 2008.

[35] P. Y. Chen, H. Huang, D. Akinwande, and A. Alù, "Graphene-based plasmonic platform for reconfigurable terahertz nanodevices," ACS Photonics, vol. 1, no. 8, pp. 647-654, Jul. 2014.

[36] S. A. Thiele, J. A. Schaefer, and F. Schwierz, "Modeling of graphene metal-oxide-semiconductor field-effect transistors with gapless large-area graphene channels," J. Appl. Phys., vol. 107, pp. 094505094505, May 2010.

[37] K. N. Parrish, and D. Akinwande, "An exactly solvable model for the graphene transistor in the quantum capacitance limit," Appl. Phys. Lett., vol. 101, pp. 053501-053501, Jul. 2012.

[38] H. Cheng, S. Chen, P. Yu, X. Duan, B. Xie, and J. Tian, "Dynamically tunable plasmonically induced transparency in periodically patterned graphene nanostrips," Appl. Phys. Lett., vol. 103, pp. 203112-203112, Nov. 2013.

[39] S. Thongrattanasiri, A. Manjavacas, and F. J. García de Abajo, "Quantum finite-size effects in graphene plasmons," ACS Nano, vol. 6, no. 2, pp. 1766-1775, Jan. 2012.

[40] H. Ren, J. Shao, M. Zhou, B. Arigong, J. Ding, and H. Zhang, "Design of dual-band transmission line with flexible phase shifts and its applications," Electron. Lett., vol. 51, no. 3, pp. 261-262, Feb. 2015.

[41] Y. S. Wong, S. Y. Zheng, and W. S. Chan, "Quasi-arbitrary phase difference hybrid coupler," IEEE Trans. Microw. Theory Tech., vol. 60, no. 6, pp. 1530-1539, Jun. 2012.

[42] S. Fratini and F. Guinea, "Substrate-limited electron dynamics in graphene," Phys. Rev. B, vol. 77, pp. 195415-195415, May 2008.

[43] S. Y. Zhou, G. -H. Gweon, A. V. Fedorov, P. N. First, W. A. De Heer, D. -H. Lee, F. Guinea, A. H. Castro Neto, and A. Lanzara, "Substrate-induced bandgap opening in epitaxial graphene," Nature Materials, vol. 6, pp. 770-775, Sep. 2007.

[44] A. Locatelli, G. Town, and C. De Angelis, "Graphene-based terahertz waveguide modulators," IEEE Trans. THz Sci. Technol., vol. 5, no. 3, pp. 351-357, May 2015. 